\title{
Avaliação do parâmetro transmissividade de LNAPLs para caracterização de áreas contaminadas no estado de São Paulo
}

\author{
Assessment of LNAPL transmissivity for contaminated \\ sites characterization in São Paulo state
}

\begin{abstract}
Marcos Vinícius Corrêa Garcia' ${ }^{\oplus}$, Ellen Caroline Puglia Leite ${ }^{1} \oplus$, Marco Aurelio Zequim Pede ${ }^{2} \oplus$,

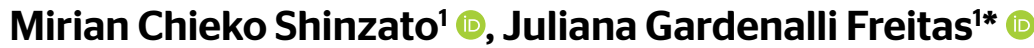

\begin{abstract}
RESUMO
Fases líquidas não aquosas leves (LNAPL), como combustíveis líquidos, estão presentes em muitas áreas contaminadas. Para o gerenciamento dessas áreas, é importante conhecer a mobilidade desse produto, a fim de subsidiar decisões sobre sistemas de remediação e avaliação de risco. Uma alternativa para quantificar a mobilidade é o parâmetro transmissividade de LNAPL, ainda pouco aplicado no Brasil. Esse trabalho avaliou a aplicabilidade desse parâmetro em diferentes áreas contaminadas por LNAPL. Foram realizados testes em três áreas com litologias, tipos de contaminantes e históricos de contaminação distintos. Em alguns testes, não foi possível determinar a transmissividade de LNAPL devido à instabilidade do nível de fluidos, por consequência da operação de sistemas de bombeamento e precipitação, além de problemas operacionais durante a realização do teste. Apesar de ser um teste simples, em alguns casos, a determinação de transmissividade de LNAPL pode ser inviável, principalmente para LNAPL de baixa mobilidade por requerer testes mais prolongados. Os principais fatores determinantes no valor de transmissividade de LNAPL foram a litologia, a posição do poço na pluma e a posição do nível d'água em relação a série histórica. Assim, a transmissividade de LNAPL pode ser um parâmetro útil no gerenciamento de áreas contaminadas, cuja interpretação deve ser feita de forma cautelosa e integrada com outros dados de investigação da área.
\end{abstract}

Palavras-chave: contaminantes orgânicos; fase livre; mobilidade; água subterrânea; monitoramento.

\begin{abstract}
Light non-aqueous phase liquids (LNAPL), such as liquid fuels, are present in many contaminated sites. To adequately manage these sites, it is important to know the product mobility, to support decisions on remediation systems and risk assessment. One alternative to quantify mobility is to quantify the LNAPL transmissivity, which is rarely applied in Brazil. This work aimed to evaluate the applicability of this parameter in LNAPL contaminated sites with different conditions. Tests were performed in three sites, with different lithologies, types of contaminants and contamination histories. In some tests, it was not possible to obtain representative values of $L N A P L$ transmissivity due to fluids levels instability, caused by pumping systems and precipitation events, and operational problems during the tests. Despite being a simple test, in some cases, the determination of LNAPL transmissivity may not be feasible, especially for low mobility LNAPLS, that require long period tests. The main factors controlling LNAPL transmissivity values were lithology, position of the well in the plume, and water level elevation in relation to the historical series. Thus, LNAPL transmissivity can be a useful parameter in the management of contaminated sites, but its interpretation must be made cautiously and integrated with other site data.
\end{abstract}

Keywords: organic contaminants; free phase; mobility; groundwater; monitoring.

\section{INTRODUÇÃO}

Historicamente, áreas contaminadas por fases líquidas não aquosas menos densas que a água (light non-aqueous phase liquids - LNAPL) representam um desafio significativo de gestão (TOMLINSON et al., 2017; EBRAHIMI et al., 2019; AZIMI et al., 2020). Os problemas mais comuns associados a LNAPLs são relacionados à liberação de produtos petrolíferos ao ambiente, seja acidentalmente ou por seu uso inadequado.

Após vazamento em subsuperfície, o LNAPL tende a migrar através da zona não saturada em fluxo descendente, deixando uma fração retida (fase residual) nos poros do solo (PARKER \& ISLAM, 2000). Enquanto houver aporte

'Departamento de Ciências Ambientais, Universidade Federal de São Paulo - Diadema (SP), Brasil.

¿Laboratório de Estudo de Bacias, Universidade Estadual Paulista - Rio Claro (SP), Brasil.

*Autor correspondente: jgfreitas@unifesp.br

Conflitos de interesse: os autores declaram não haver conflitos de interesse.

Financiamento: Conselho Nacional de Desenvolvimento Científico e Tecnológico (CNPq), processo 426953/2016-9.

Recebido: 11/06/202O - Aceito: 03/12/2020 - Reg. ABES: 20200214 
suficiente, o LNAPL continuará migrando até encontrar o topo da zona saturada (franja capilar), onde, devido à sua menor densidade, passa a migrar lateralmente como uma fase contínua e móvel (fase livre), causando uma depressão da franja capilar e zona saturada (NEWELL et al., 1995; TOMLINSON et al., 2017; EBRAHIMI et al., 2019). A distribuição do LNAPL em profundidade depende das propriedades do meio (como textura, porosidade e pressão capilar), das propriedades da fase líquida não aquosa (non-aqueous phase liquids (NAPL)) (como densidade, molhabilidade e viscosidade) e do volume liberado (NEWELL et al., 1995; CHARBENEAU et al., 2000; API, 2003; JENSEN; FALTA, 2005; ILLANGASEKARE et al., 2005; TOMLINSON et al., 2017; EBRAHIMI et al., 2019). A presença simultânea de duas fases líquidas promove uma competição pelo espaço do poro e, como resultado, a mobilidade de cada fase diminui, sendo variável em função de suas respectivas saturações (NEWELL et al., 1995; WINEGARDNER; TESTA, 2000; JENSEN; FALTA, 2005).

No levantamento realizado pela CETESB (2019), 71\% (4.475) das áreas contaminadas e reabilitadas no Estado de São Paulo são postos de combustíveis com contaminação relacionada aos LNAPLs, cuja remediação utilizada foi, predominantemente, por bombeamento. No entanto, essa técnica apresenta baixa efetividade em razão da redução da saturação de NAPL e, consequentemente, da sua mobilidade ao longo da remediação (OOSTROM et al., 2005). Conhecer como o LNAPL está presente no meio, sua mobilidade e o potencial de recuperação da fase livre permite realizar uma análise crítica entre os esforços demandados nessa técnica de remediação e a sua eficiência para a proteção ambiental; além de planejar uma melhor estratégia de gerenciamento (BECKETT et al., 1997; AZIMI et al., 2020).

A mobilidade do LNAPL pode ser determinada pela transmissividade do LNAPL (Tn), que quantifica a capacidade da massa de LNAPL mover-se no meio poroso ou fraturado sob condições de saturação mista (BECKETT \& HUNTLEY, 2015; KIRKMAN \& KOONS, 2020). Trata-se de uma métrica resumida que incorpora propriedades do aquífero e propriedades físicas, saturação e permeabilidade relativa do NAPL (HAWTHORNE \& KIRKMAN, 2011; ASTM, 2013). Assim, a Tn correlaciona-se com a capacidade de recuperação do LNAPL, fornecendo um parâmetro comparável entre locais, sendo uma medida substancialmente melhorada em relação à espessura aparente de NAPL nos poços (HAWTHORNE \& KIRKMAN, 2011; EBRAHIMI et al., 2019). A determinação e a interpretação desse parâmetro são complexas, principalmente por não ser uma constante da formação e apresentar imprecisões, dependendo das condições da área, como espessura da franja capilar e viscosidade do produto (HUNTLEY \& BECKETT, 2002; ITRC, 2018; AHMED et al., 2019). Ela pode variar de acordo com a metodologia aplicada, condições e duração do teste, mudanças nas condições piezométricas, propriedades dos fluidos e condições em que o LNAPL se encontra em subsuperfície (HAWTHORNE \& KIRKMAN, 2011; SIMON, 2012; GATSIOS et al., 2018; ATTEIA et al., 2019).

A determinação de Tn pode ser uma ferramenta nas tomadas de decisões, auxiliando no planejamento de testes-piloto de recuperação de LNAPL e na otimização de soluções de remediação (LENHARD et al., 2017; ITRC, 2018; SOOKHAK LARI et al., 2020). Em alguns locais, pode servir como métrica para encerramento da remediação, tendo sido adotada por alguns órgãos reguladores, como a Agência de Proteção Ambiental dos Estados Unidos da América (USEPA) (SIMON, 2012; KIMBALL et al., 2018). A Interstate Technology and Regulatory Council (ITRC) propôs em 2009 para os EUA uma faixa de referência de aproximadamente 0,0093 a $0,074 \mathrm{~m}^{2} \mathrm{dia}^{-1}$ como mínimo indicativo do potencial de recuperação de produto em fase livre. Os Estados de Michigan e Nebraska adotaram como indicativo do potencial de recuperação valores de Tn de $0,046 \mathrm{~m}^{2} \mathrm{dia}^{-1}$, enquanto no Estado de Massachusetts o valor limite de taxa de recuperação de LNAPL adotado foi de $0,074 \mathrm{~m}^{2} \mathrm{dia}^{-1}$ (KIMBALL et al., 2018).

Considerando o grande número de áreas contaminadas por LNAPL no Estado de São Paulo e as particularidades dos contaminantes no Brasil, a Tn pode ser um parâmetro de grande utilidade para diagnóstico e gestão dessas áreas. No entanto, esse tipo de teste raramente é realizado, e a sua aplicabilidade nas condições brasileiras ainda não foi avaliada. Diante desse contexto, o presente trabalho teve como objetivo avaliar o método de quantificação da transmissividade de LNAPL, considerando diferentes condições ambientais e grau de contaminação, buscando subsidiar a aplicação desse parâmetro na gestão de áreas contaminadas. Dessa forma, procurou-se identificar as condições das áreas que dificultam ou limitam a aplicação dessa metodologia e discutir o seu uso no gerenciamento de áreas com LNAPL.

\section{METODOLOGIA}

\section{Áreas de estudo}

Os testes de transmissividade de LNAPL foram realizados entre outubro de 2016 e março de 2017 em três áreas distintas (Figura 1). Informações sobre as áreas foram obtidas de relatórios de investigação feitos por empresas de consultoria e fornecidos pelos responsáveis pelas áreas durante as atividades de campo.

\section{Área 1}

Comércio varejista de combustível localizado no município de Ribeirão Pires, São Paulo. Encontra-se no Embasamento Cristalino onde ocorrem gnaisses intemperizados de coloração marrom acastanhada, gerando saprólitos arenosos e/ou argilosos (IPT, 2000). No local dos poços de monitoramento ocorrem material de aterro de textura siltosa sobre outro material silte-arenoso (com cerca de $3 \mathrm{~m}$ de espessura), onde se encontra o nível dágua. Abaixo há um material mais grosseiro (cascalho), que se encontra próximo à rocha sã (gnaisse). O local apresenta contaminação por vazamento de um tanque de armazenamento de gasolina, e tem sido realizado o bombeamento de fase livre desde 2015. O teste de transmissividade foi realizado em um único poço de monitoramento (PM) (Figura 2).

\section{Área 2}

Garagem de ônibus localizada no município de São Paulo. Encontra-se na transição da Bacia Sedimentar de São Paulo e o Embasamento Cristalino. De acordo com o relatório de investigação da área, o solo é homogêneo e apresenta textura silte-arenosa de coloração variegada (podendo indicar os sedimentos típicos da Bacia Sedimentar de São Paulo). A condutividade hidráulica (K) no local tem valor médio de $7,33 \times 10^{-5} \mathrm{~cm} \mathrm{~s}^{-1}$, e a velocidade de fluxo foi estimada em $11,13 \mathrm{~m} \mathrm{ano}^{-1}$. A contaminação da área ocorreu devido ao vazamento de uma linha que conecta o tanque de armazenamento de diesel com os bicos de abastecimento. A remediação da área está sendo realizada por sistema de bombeamento em operação há um ano. Os testes de Tn nessa área foram realizados em dois poços de monitoramento (PM-02 e PM-17) (Figura 2). 


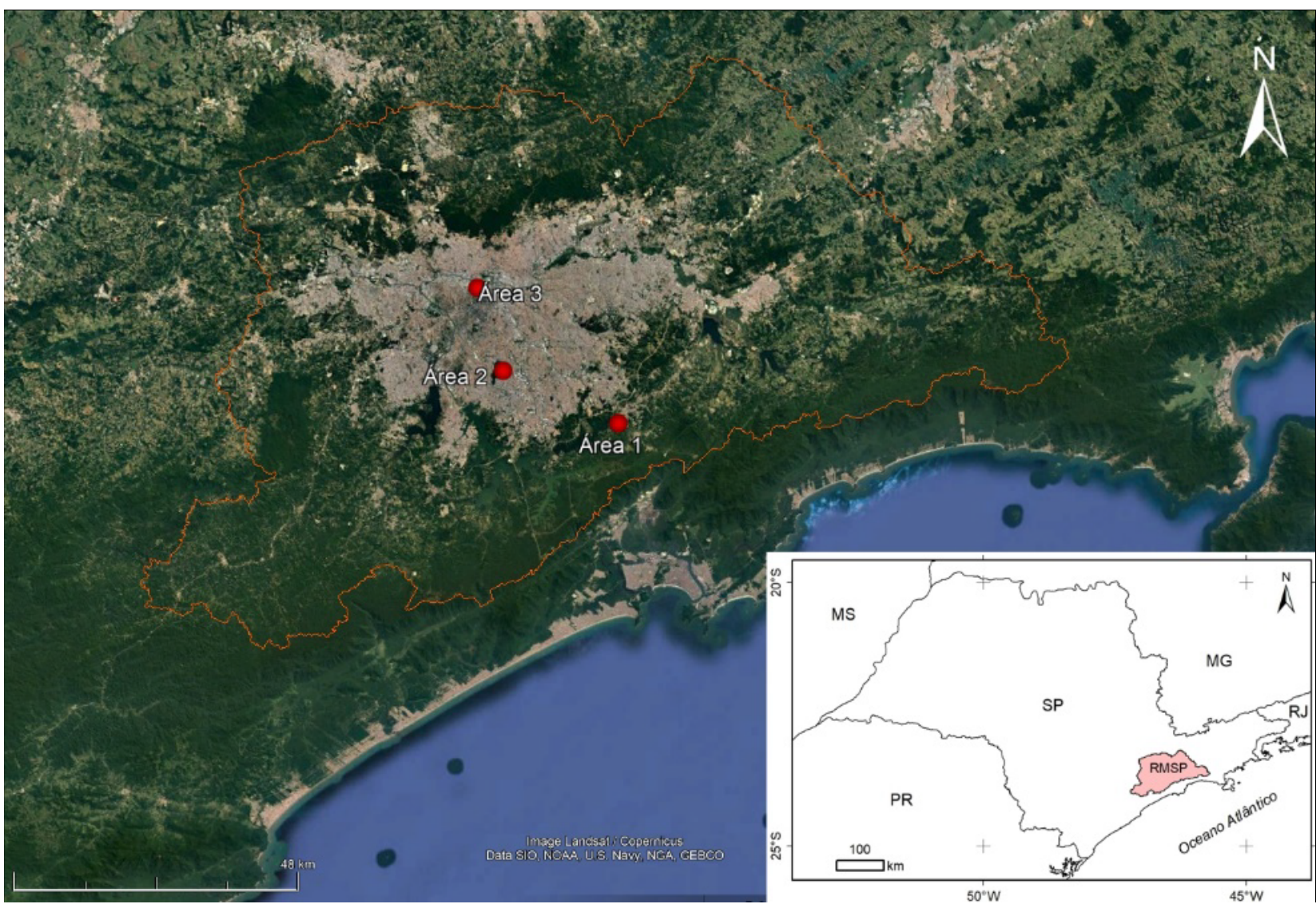

Fonte: Google, 2020.

Figura 1 - Localização das áreas de estudo.

\section{Área 3}

Garagem de ônibus desativada no município de São Paulo. No local, há depósitos aluviais da bacia hidrográfica do Alto Tietê, que ocorre na Bacia Sedimentar de São Paulo. A área foi contaminada durante o funcionamento de uma empresa de transporte, na década de 1940, que realizava manutenção e abastecia a frota de ônibus, além de armazenar combustível e resíduos de óleo. Lá existe uma pluma de fase livre de óleo diesel, avaliada em testes de Tn nos poços PM-22 e SBF-03. Por se tratar de um terreno vazio, foi possível realizar uma sondagem a trado manual para descrever a litologia. No poço PM-22, observou-se uma camada de aterro de $0,5 \mathrm{~m}$, seguida de camadas de solo argilo-siltoso de cor verde (1 m) e solo argiloso de cor preta (cerca de $1 \mathrm{~m}$ de espessura). Abaixo dessa camada ocorre um solo arenoso de coloração de cinza ( $1 \mathrm{~m}$ de espessura), seguido de outro arenoso mais grosso (Figura 2). Estudos anteriores indicam que a $\mathrm{K}$ média na área é de $1,26 \times 10^{-4} \mathrm{~cm} \mathrm{~s}^{-1}$ e a velocidade de fluxo é $41,47 \mathrm{~m} \mathrm{ano}^{-1}$.

\section{Testes para determinação da transmissividade de LNAPL}

Foram realizados nove testes de Tn em cinco poços de monitoramento distintos. Os poços foram selecionados em função da presença e posição da fase livre, garantindo que os níveis de água e óleo estavam posicionados na seção filtrante do poço ao longo de todo o teste. Para a realização dos testes de Tn foi utilizado o método Baildown, que consiste na remoção da fase livre de LNAPL do poço de monitoramento e no registro da variação dos níveis de água e óleo em função do tempo (ASTM, 2013). O volume de LNAPL a ser removido foi previamente determinado pela Equação 1, a fim de evitar a influência do acúmulo de produto no pré-filtro (ASTM, 2013).

$V_{t}=V_{c}+V_{b}=b_{n} \pi r_{c}^{2}+S_{y f} b_{b} \pi\left(r_{b}^{2}-r_{c}^{2}\right)$

Em que: $\mathrm{V}_{\mathrm{t}}$ : volume total efetivo de LNAPL no furo de sondagem $\left[\mathrm{L}^{3}\right] ; \mathrm{V}_{\mathrm{b}}$ : volume de LNAPL no pré-filtro $\left[\mathrm{L}^{3}\right] ; \mathrm{V}_{\mathrm{c}}$ : volume de LNAPL no poço $\left[\mathrm{L}^{3}\right] ; \mathrm{b}_{\mathrm{b}}$ : espessura de LNAPL no pré-filtro [L]; $b_{n}$ : espessura do LNAPL medido [L]; $r_{c}$ : raio do poço $[\mathrm{L}] ; \mathrm{r}_{\mathrm{b}}$ : raio do furo de sondagem $[\mathrm{L}] ; \mathrm{S}_{\mathrm{yf}}$ coeficiente de armazenamento do filtro, adotado igual 0,190 (ASTM, 2013).

A remoção do LNAPL foi realizada com amostradores de água subterrânea descartáveis de polietileno (bailer), e o volume retirado foi medido em uma proveta graduada. Ao atingir o volume calculado, iniciaram-se as medidas das profundidades dos níveis de LNAPL (NO) e água (NA) com medidor tipo interface (Heron Instruments, modelo H.OIL), até que esses dois parâmetros atingissem valores próximos aos iniciais. Os dados de NO e NA foram 


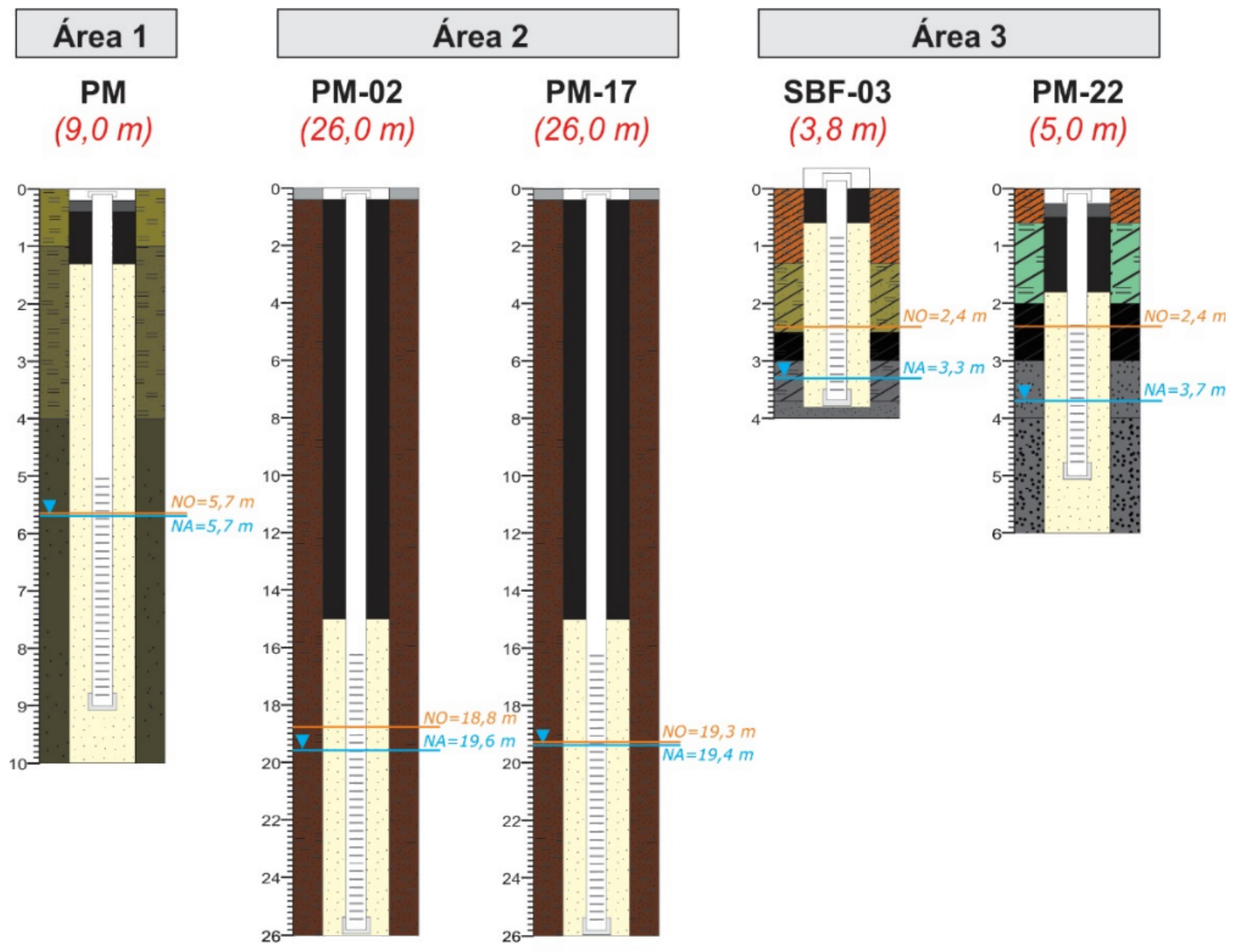

\section{Legenda}

Aterro de argila, silto arenosa, amarela, cinza e vermelha

Silte arenoso, coloração variegada

Argila siltosa, pouco arenosa, amarela e cinza escura

Argila areno siltica, marrom

Aterro de entulho

Aterro siltoso

Areia grossa, cinza

Sedimento de silte arenoso

Pré-filtro
Argila orgânica, silto arenosa, preta

Argila siltosa pouco arenosa, cinza

Areia fina, pouco argilosa, cinza

Argila preta

Areia média, cinza

Aterro de argila, areno síltica

Argila síltica, verde

Cascalho

Bentonita

NA: nível de água; NO: nível de óleo; PM: poço de monitoramento. Nível de água e óleo representados são os medidos no primeiro teste de transmissividade realizado no poço. Fonte: elaborada pelos autores. 
registrados a cada 1 minuto durante os primeiros 30 minutos do teste. Em seguida, a frequência dos registros foi alterada de acordo com o andamento do teste em cada área de estudo.

\section{Caracterização dos LNAPLs}

Foram coletadas amostras de LNAPL para determinação de viscosidade e densidade em laboratório. A densidade foi obtida pela razão entre a massa e o volume de NAPL. A massa correspondente a $100 \mu \mathrm{L}$ de LNAPL foi determinada em uma balança semianalítica (BL320H/Shimadzu) com o auxílio de uma seringa. As viscosidades relativas das amostras $\left(\mu_{\text {ree }}\right)$ foram determinadas em um viscosímetro de Ostwald (ALMEIDA et al., 1995). Todas as determinações foram feitas à temperatura de $21^{\circ} \mathrm{C}$.

\section{Análise de dados}

Para estimar Tn a partir dos dados obtidos em campo, foram aplicados os modelos de Bouwer e Rice (1976) e de Cooper e Jacob (1946), utilizando as planilhas desenvolvidas pelo Instituto Americano do Petróleo (API, 2016). O método de Bouwer e Rice (B\&R) aplica um modelo linear (equação de Thiem) que relaciona a descarga de LNAPL do meio com o valor do rebaixamento do nível de LNAPL, baseado na continuidade do volume de LNAPL dentro do poço. Esse método considera o logaritmo da variação do rebaixamento como uma função linear do tempo. O valor de Tn por esse método é determinado através da relação da inclinação de uma reta ajustada. O modelo de Cooper e Jacob (C\&J) também considera o fluxo e o rebaixamento do LNAPL, mas em função do tempo, e necessita que o coeficiente de armazenamento seja estimado. Os parâmetros de transmissividade e armazenamento do LNAPL são estimados por meio da comparação entre os valores de descarga verificados em campo e os valores gerados através do modelo numérico (API, 2016).

Os valores de Tn foram avaliados em relação às propriedades físicas da área (litologia e profundidade do nível d’água) e do LNAPL (densidade e viscosidade), além de outras condições específicas encontradas em cada área (sistema de bombeamento em andamento, quantidade e estado dos poços, eventos de chuva etc.). Testes realizados no mesmo poço foram utilizados para avaliar o impacto da diferença da posição do nível d’água e da forma de realização do teste. O efeito da posição do poço na pluma foi avaliado nos testes realizados em poços de monitoramento distintos, mas instalados no mesmo local. A comparação entre áreas distintas permitiu avaliar o efeito das propriedades analisadas na determinação de Tn.

\section{RESULTADOS E DISCUSSÃO}

Os parâmetros analisados e determinados em cada poço de monitoramento por área encontram-se resumidos na Tabela 1. Em dois testes (2 e 5), não foi possível efetuar a determinação de Tn, pois as curvas apresentaram comportamentos diferentes do previsto pelo modelo. Duas principais razões foram identificadas: ocorrência de níveis não estáticos e não atendimento das condições ideais para a realização dos testes. Os valores de Tn foram nulos em seis testes e mais elevados em dois (testes 6 e 7 no PM-22/área 3). Somente nesse poço os valores de Tn foram indicativos de NAPL com mobilidade e potencial de migração (BECKETT \& LUNDEGARD, 1997; ITRC, 2009). Verificou-se que as principais variáveis que interferiram no valor de Tn foram aquelas relacionadas à litologia da área e posição do nível dágua. A interferência das principais variáveis e/ou condições e considerações sobre os testes de transmissividade são analisadas nos itens seguintes.

\section{Efeitos dos níveis não estáticos previamente à realização dos testes}

Após a remoção de NAPL, inicialmente ocorre uma elevação do NA, que foi observada na maioria dos testes nos primeiros instantes de monitoramento, decorrente do alívio de pressão causado pela remoção do LNAPL. Na sequência, é esperado o aporte de NAPL para dentro do poço, resultando em diminuição do NA até valores próximos aos iniciais (ASTM, 2013). Esse comportamento foi observado claramente no teste 7 (Figura 3D). No entanto, observou-se em alguns testes desvio desse comportamento em razão das flutuações do nível

Tabela 1 - Descritivo dos testes e sumário dos resultados obtidos.

\begin{tabular}{|c|c|c|c|c|c|c|c|c|c|c|}
\hline \multirow{2}{*}{\multicolumn{2}{|c|}{ Teste / poço }} & \multicolumn{3}{|c|}{ Condições iniciais } & \multicolumn{2}{|c|}{ Propriedades do NAPL } & \multicolumn{4}{|c|}{ Teste de Tn } \\
\hline & & $\mathrm{NO}(\mathrm{m})$ & $\mathrm{NA}(\mathrm{m})$ & $\begin{array}{l}\text { Espessura da } \\
\text { fase livre }(m)\end{array}$ & $\begin{array}{l}\text { Densidade } \\
\left(\mathrm{g} \mathrm{mL}^{-1}\right)\end{array}$ & $\begin{array}{l}\text { Viscosidade } \\
\text { relativa }\end{array}$ & $\begin{array}{l}\text { Data de } \\
\text { início }\end{array}$ & $\begin{array}{c}\text { Duração } \\
\text { (min) }\end{array}$ & $\begin{array}{c}\text { Fase livre } \\
\text { recuperada (\%) }\end{array}$ & $\begin{array}{c}\text { Tn } \\
\left(m^{2} d^{-1}\right)\end{array}$ \\
\hline \multicolumn{11}{|c|}{ Área 1} \\
\hline 1 & PM & 5,735 & 5,770 & 0,035 & 0,86 & 6,59 & 23/11/16 & 8349 & 94 & O \\
\hline \multicolumn{11}{|c|}{ Área 2} \\
\hline 2 & \multirow{3}{*}{$\mathrm{PM}-\mathrm{O} 2$} & 18,820 & 19,600 & 0,220 & 0,83 & 3,60 & 07/10/16 & 61 & - & * \\
\hline 3 & & 20,028 & 20,310 & 0,282 & 0,84 & 3,66 & 29/10/16 & 406 & 37 & 0 \\
\hline 4 & & 20,048 & 20,080 & 0,032 & 0,81 & 3,50 & 08/02/17 & 70 & 62 & 0 \\
\hline 5 & PM-17 & 19,365 & 19,435 & 0,07 & 0,84 & 3,40 & 21/11/16 & 6090 & 50 & * \\
\hline \multicolumn{11}{|c|}{ Área 3} \\
\hline 6 & \multirow{2}{*}{ PM-22 } & 2,428 & 3,762 & 1,334 & 0,82 & 7,31 & 12/12/16 & 15593 & 92 & 0,082 \\
\hline 7 & & 2,503 & 3,805 & 1,302 & 0,85 & 5,17 & 09/03/17 & 127113 & 95 & 0,122 \\
\hline 8 & \multirow{2}{*}{ SBF-O3 } & 2,452 & 3,340 & 0,888 & 0,84 & 7,90 & 12/12/16 & 14428 & 30 & 0,003 \\
\hline 9 & & 2,517 & 3,905 & 0,578 & 0,84 & 5,41 & 09/03/17 & 126912 & 37 & 0,005 \\
\hline
\end{tabular}

*Não determinada. PM: poço de monitoramento; NA: nível de água; NO: nível de óleo; NAPL: fase líquida não aquosa; Tn: parâmetro de transmissividade de LNAPL.

Fonte: elaborada pelos autores. 

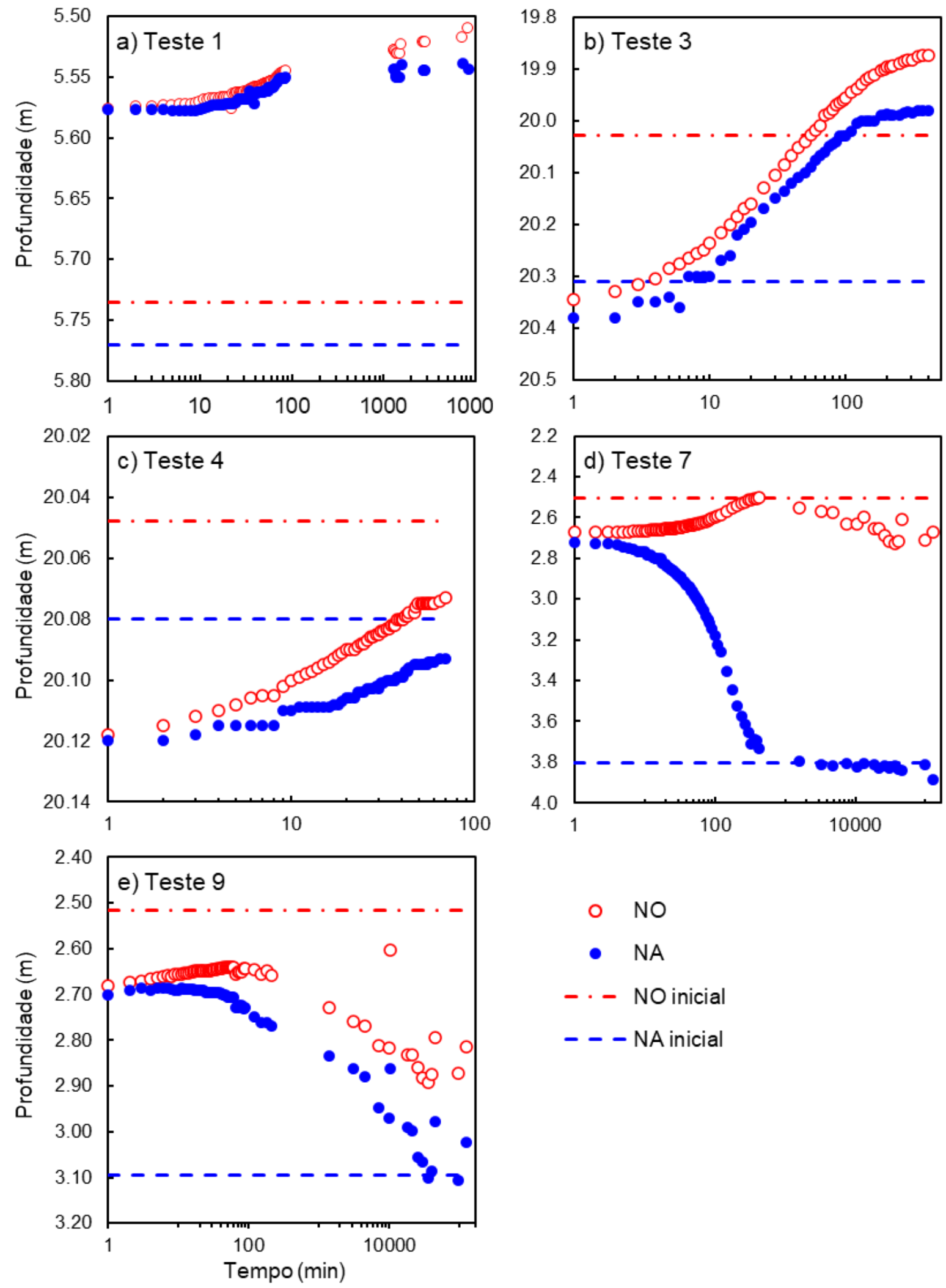

$$
\begin{gathered}
0 \text { NO } \\
-N A \\
-\cdots \text { NO inicial } \\
-- \text { - NA inicial }
\end{gathered}
$$


d’água por fatores externos. Por exemplo, no teste 1, observou-se uma elevação constante do NA (Figura 3A) devido à influência das chuvas que ocorreram na área 1 (que possui lençol freático raso e alta K. Assim, a elevação do NA observada é uma resposta aos eventos de precipitação, e o valor de Tn calculado não é representativo da área. $O$ efeito da precipitação também foi observado no teste 6 . A curva de recuperação de fase livre desse teste foi muito similar ao teste 7 , realizado no mesmo poço, mas ocorreu uma redução da espessura de fase livre em períodos de chuva (Figura 4B) e consequente redução do valor de Tn (Tabela 1). Portanto, em locais com lençóis freáticos mais rasos, é indicado que os testes de Tn sejam realizados em períodos secos, para evitar que o NA esteja sujeito a variações provocadas pelas precipitações.

$\mathrm{Na}$ área 2 (testes 2 a 5) também foi observada variação dos níveis em razão de variáveis externas, nesse caso a presença de um sistema de remediação por bombeamento. $\mathrm{O}$ teste 2 foi realizado com o sistema em funcionamento, o teste 3 uma hora após o desligamento do sistema, e o teste 4, três dias após o desligamento. O teste 5 foi realizado com o sistema em operação, mas o poço se localizava fora da zona de influência do sistema. Nos testes 3 e 4, observou-se que o período entre o desligamento das bombas e o início dos testes não foi suficiente para a estabilização dos níveis. Apesar das avaliações durante a caracterização das condições iniciais do poço de monitoramento indicarem níveis estáveis, foi observada a elevação do NA durante o período monitorado (Figura 3B e 3C) em decorrência do desligamento do sistema de remediação. Portanto, é necessário acompanhar a variação dos NA e NO por um longo período para evitar que os testes sejam iniciados antes de sua estabilização. Outro efeito observado pelo desligamento do sistema de bombeamento e consequente elevação do NA foi a conversão da fase livre em fase residual, reduzindo a espessura de fase livre no poço no teste 4 em relação ao teste 3 (Figura $4 \mathrm{~A}$ ).

No teste 9 (Figura 3E), observou-se um comportamento distinto, mas também provavelmente afetado por níveis não estáticos causados por variáveis externas. Até aproximadamente $100 \mathrm{~min}$, as curvas se comportaram como esperado, mas depois desse período a profundidade dos NA e NO aumentaram com o tempo. Essa queda pode estar associada a variações regionais do nível d'água ou ao funcionamento de sistemas de bombeamento externos à área de estudo.

Garantir a estabilidade e o equilíbrio dos níveis pode ser extremamente complexo em áreas que possuem sistema de remediação ativo ou muito sujeitas à influência de chuva. Como o tempo demandado para estabilização dos níveis e determinação de Tn pode ser muito longo, esse teste pode ser inadequado para algumas áreas por deixar o sistema de remediação inoperante. Além disso, em alguns testes de duração prolongada (mais de dois meses), como aqueles com baixos valores de Tn, é muito difícil que os níveis não sejam afetados por outros fatores, como precipitação e sistemas de bombeamento externos à área.

\section{Efeitos dos procedimentos de campo/operacionais}

Em testes com pequena espessura de LNAPL, a aplicação da técnica de baildown é prejudicada pela dificuldade em remover o LNAPL do poço sem causar alteração no nível d'água. Apesar de relativamente simples, a remoção de LNAPL com bailer pode trazer também parte da água que se encontra junto ao produto oleoso. Alguns testes realizados (resultados não apresentados) mostraram que o uso de bomba peristáltica pode minimizar esse problema, como realizado em Palmier et al. (2016). A remoção de água altera o equilíbrio no poço de monitoramento, interferindo no resultado do teste. Esse efeito pode ser visto nas medições do teste 4, na área 2 (Figura 3C), que tinha uma espessura inicial de LNAPL de 3,2 cm. A remoção de água com o NAPL causou um rebaixamento do nível d'água, que aparece com as curvas se iniciando abaixo do nível d'água inicial. Essa dificuldade é reconhecida na norma E2856-13 da $\operatorname{ASTM}$ (2013), que recomenda que em poços de monitoramento com espessuras de LNAPL menores que 0,152 m não seja utilizada a técnica de baildown. Nos testes em que ocorreu esse problema, observou-se uma maior recuperação de água nos poços quando comparada ao volume de LNAPL, impossibilitando a
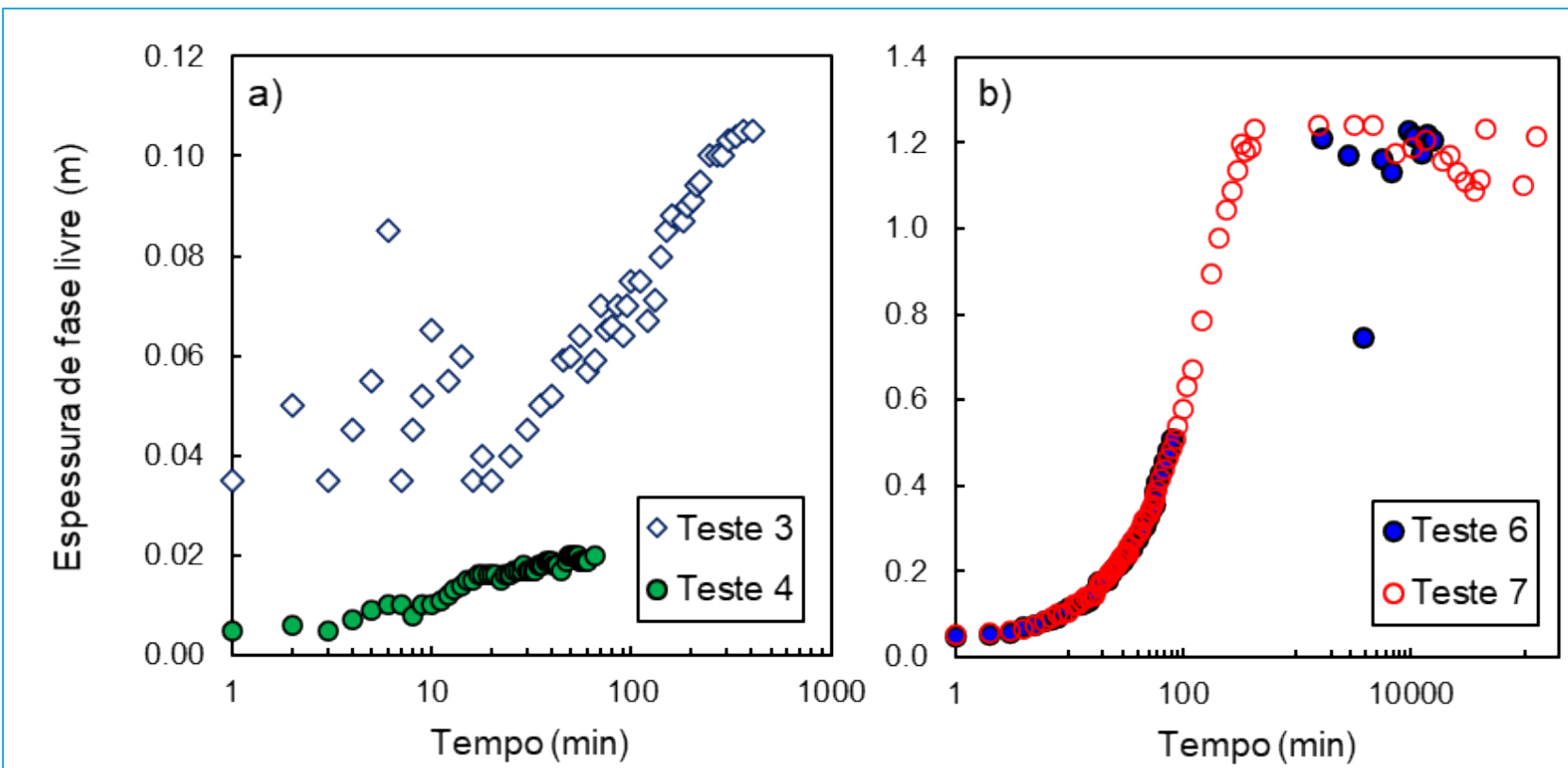

Fonte: elaborada pelos autores.

Figura 4 - Recuperação da espessura de fase livre ao longo dos testes 3 e 4 (A) e 6 e 7 (B). 
determinação de Tn. A medição das interfaces ar-óleo e óleo-água com equipamentos manuais também pode ter gerado imprecisões, dificultando a interpretação, principalmente quando as variações de nível são pequenas, como destacado por Palmier et al. (2016).

A localização e o estado de conservação do poço de monitoramento também interferiram nos valores de Tn. Foi observado no trabalho de campo que um dos poços escolhido para ser monitorado (PM-17 da área 2) recebia periodicamente aporte superficial de água de lavagem de veículos contendo óleo e detergente, como evidenciado pela presença de material oleoso no fuso do cap e flotação de LNAPL no líquido presente na câmara de calçada do poço de monitoramento, após atividades de lavagem dos veículos. A entrada dessa parcela de fluido interferiu nos níveis de NA e NO, gerando resultados não representativos.

\section{Propriedades determinantes para transmissividade de LNAPL}

Com base nas observações dos testes nas diferentes áreas, foram identificadas as principais variáveis que contribuíram para obtenção de valores mais altos de Tn. Essas propriedades estão relacionadas às características físicas da área (litologia), posição dos poços em relação à pluma e à elevação do NA e sua relação com a série histórica, conforme detalhado na sequência.

As áreas 1, 3 e 4 encontram-se sobre aterros com características físicas muito heterogêneas e distintas das formações originais. $\mathrm{Na}$ área 3, apesar do PM-22 e do SBF-03 estarem inseridos na mesma pluma de LNAPL, o SBF-03 apresentou valores menores de Tn (Tabela 1). Considerando que as propriedades do produto (viscosidade, densidade e composição) são praticamente as mesmas, a diferença dos valores de Tn provavelmente está associada ao meio visto que no SBF-03 o LNAPL encontra-se na camada de argila siltosa pouco arenosa e no PM-22 em areia média. Outro fator que pode ter influenciado no valor de Tn é a localização na pluma, já que o PM-22 estava situado no centro da pluma enquanto o SBF-03 na borda, com menor espessura de fase livre.

Todas as amostras de LNAPL apresentaram valores de densidade semelhantes $\left(0,84 \pm 0,01 \mathrm{~g} \mathrm{~mL}^{-1}\right)$. Quanto à viscosidade relativa, os produtos analisados nas áreas 1 e 3 apresentaram valores mais altos (de 5,4 a 7,3) em relação às áreas $2 \mathrm{e} 4$ (em torno de 3,5). Apesar dos contaminantes das áreas 2 e 3 terem sido identificados como óleo diesel, a diferença nos valores de viscosidade pode estar relacionada à composição e à idade do produto, visto que a contaminação da área 3 é da década de 1970, enquanto nas demais áreas a contaminação é mais recente. Considerando que a $\mathrm{K}$ é função das propriedades do meio e do fluido (Equação 2), as variações observadas resultam em mobilidade até duas vezes superior para o produto da área 2 em relação ao produto da área 3 . No entanto, essa diferença não resultou em maiores valores de Tn na área 2, indicando que esse não foi um fator determinante. Todavia, em áreas onde o NAPL contém características muito distintas (como óleo de motor, creosoto), a relevância desses parâmetros se torna maior.

$\mathrm{K}=\frac{\mathrm{k} \rho \mathrm{g}}{\mu}$

Em que: k: permeabilidade do meio; $\rho$ : densidade do fluido; g: aceleração da gravidade; $\mu$ : viscosidade do fluido.

Outro aspecto importante refere-se à posição do lençol freático no momento do teste e em relação à série histórica. A importância da posição do NA também foi identificada em estudos usando modelos matemáticos (LENHARD et al., 2017). Esse efeito pode ser observado nos resultados dos poços PM-22 e SBF-03, onde foram determinados maiores valores de Tn quando o NA estava mais profundo (Tabela 1). Esse comportamento é esperado, pois, quando o NA está em posições mais elevadas, uma fração maior do produto fica imobilizada como fase residual na zona saturada. Quando o nível d'água diminui, parte desse produto volta para a fase livre, aumentando a mobilidade do produto (ILLANGASEKARE et al., 2005). Assim, é de grande importância que sejam realizados monitoramentos da espessura de fase livre por períodos longos e a Tn seja avaliada nesse contexto, como destacado por Atteia et al. (2019).

\section{Considerações sobre o cálculo da transmissividade de LNAPL}

A determinação da Tn pode ser feita de acordo com os modelos de B\&R e C\&J (API, 2016). Apesar de os valores de Tn terem sido calculados pelos dois métodos, nesse trabalho optou-se por utilizar o método de B\&R. De forma similar ao observado por Palmier et al. (2016; 2017), os resultados de Tn obtidos pelos dois modelos foram próximos (Tabela 2), tendo o modelo de B\&R apresentado valores um pouco maiores em relação ao modelo de C\&J, com maior diferença para maiores Tn. O coeficiente de correlação $\left(R^{2}\right)$ entre os resultados dos dois métodos foi de 0,984 , indicando que a tendência dos resultados se mantém independentemente do método aplicado para o cálculo.

De acordo com a norma ASTM (2013), os testes devem ser monitorados no mínimo até se atingir $75 \%$ da condição inicial dos níveis dos fluidos. Para avaliar a importância de se atingir essa recuperação na quantificação de Tn, o cálculo do teste 8 foi refeito utilizando-se somente os dados dos primeiros 180 minutos, quando a recuperação foi de aproximadamente $20 \%$. Nesse caso, obteve-se o valor de $0,003 \mathrm{~m}^{2} \mathrm{~d}^{-1}$ pelo método B\&R. Por outro lado, utilizando-se $100 \%$ dos dados (aproximadamente dez dias), o valor de Tn obtido pelo mesmo método foi nulo. Assim, verifica-se que em áreas onde a transmissividade apresenta valores baixos, se o monitoramento for interrompido antes da recuperação de $75 \%$ do valor inicial da fase livre, apesar de não se obter o valor exato de $\mathrm{Tn}$, já se obtém a informação de que a transmissividade é bastante baixa, o que é uma informação útil para o gerenciamento da área e definição de estratégias.

\section{Síntese sobre a aplicação de LNAPL}

A análise dos resultados dos testes realizados nesse estudo permitiu distinguir duas situações relativas à aplicação de $\mathrm{Tn}$, que envolvem a possibilidade ou não de se determinar esse parâmetro (Figura 5). Nos casos em que não foi possível quantificar a Tn, as curvas de recuperação desviaram do comportamento ideal

Tabela 2- Comparação dos métodos de cálculo de transmissividade de LNAPL $\left(\mathrm{m}^{2} \mathrm{~d}^{-1}\right)$.

\begin{tabular}{l|c|c|c} 
Teste & Bouwer \& Rice & Cooper \& Jacob & Média \\
\hline Teste 6 & 0,082 & 0,07 & 0,075 \\
\hline Teste 7 & 0,122 & 0,086 & 0,104 \\
\hline Teste 8 & 0,003 & 0 & 0,0015 \\
\hline Teste 9 & 0,005 & 0,008 & 0,0065 \\
\hline
\end{tabular}

Fonte: elaborada pelos autores 


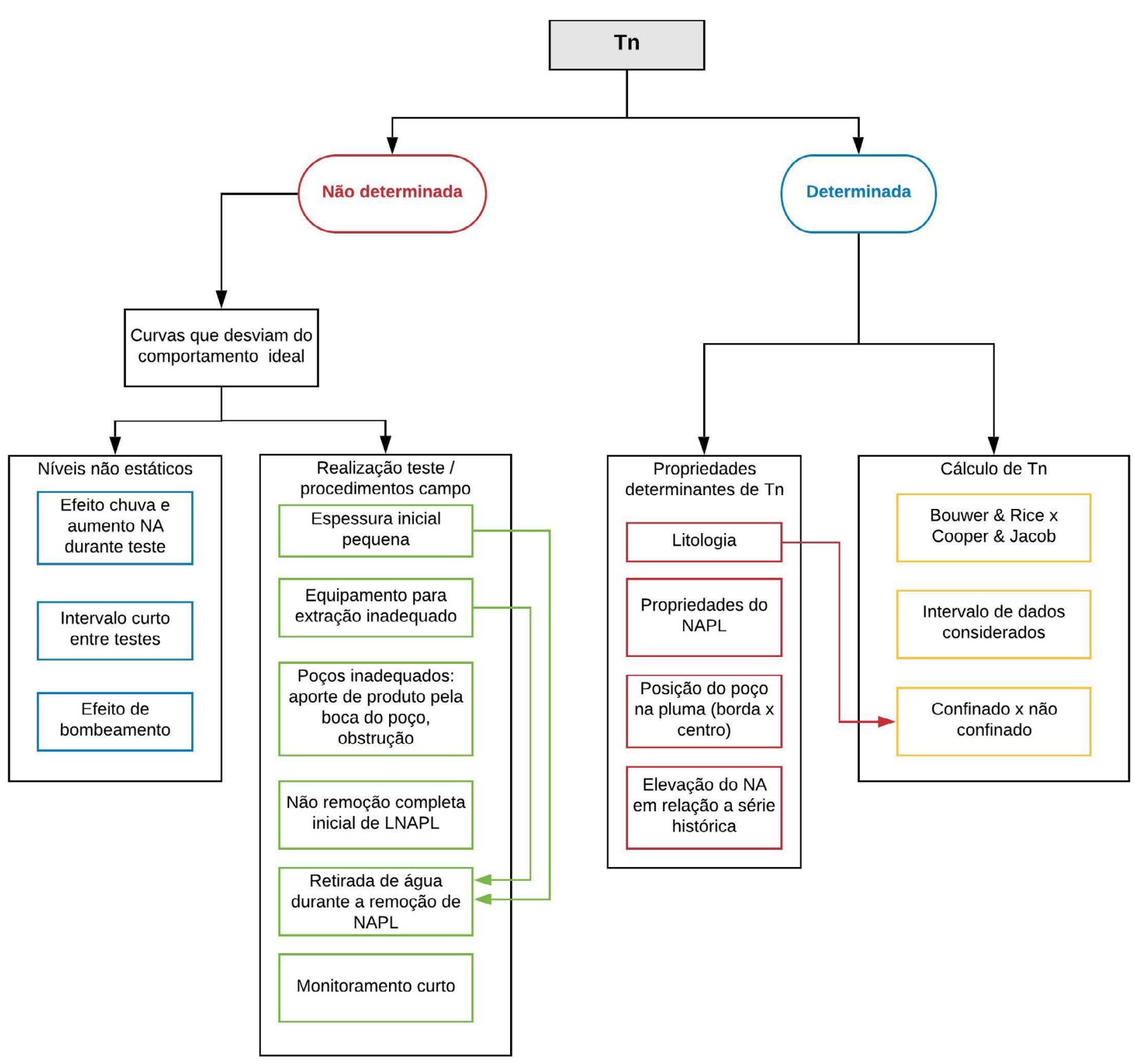

Tn: parâmetro de transmissividade de LNAPL

Fonte: elaborada pelos autores.

Figura 5 - Sumário dos principais parâmetros que interferem na determinação de transmissividade de LNAPL.

devido a dois motivos: (i) ocorrência de níveis não estáticos causados por precipitação, bombeamento ou outros fatores; e, (ii) problemas operacionais associados à realização do teste. A influência desses fatores nos testes de Tn pode ser removida ou minimizada com um planejamento adequado e conhecimento prévio da área. No entanto, considerando que alguns testes podem demandar longos períodos, pode ser impossível garantir as condições ideais. Nos locais com condições de se determinar a Tn, destacam-se três fatores que podem interferir no seu valor: a litologia da área; a posição do poço na pluma de fase livre; e a elevação do NA em relação ao nível histórico.

\section{CONCLUSÕES}

Os testes de Tn realizados em áreas com diferentes características permitiu verificar que, apesar de serem relativamente simples, demandam planejamento, definição clara dos objetivos e avaliação prévia das variáveis que podem influenciar nos seus resultados.

As principais variáveis identificadas nesse trabalho que influenciaram na determinação da Tn são a litologia, a saturação do LNAPL, a localização do poço de monitoramento na pluma de fase livre e a flutuação do nível do lençol freático. As variações de densidade e viscosidade do NAPL não foram determinantes 
nas áreas estudadas, provavelmente pelos valores terem sido relativamente próximos, mas outros produtos podem apresentar propriedades bastante distintas. Foi verificado que a estabilidade dos níveis dos fluidos é fundamental para que seja possível quantificar a Tn, sendo recomendável que os testes de Tn sejam realizados em períodos de baixa precipitação pluviométrica. A seleção dos poços para a realização do teste também deve levar em consideração a integridade construtiva, a posição do filtro, a descrição adequada do perfil construtivo e litológico, e a posição dentro da pluma de fase livre.

O conhecimento da litologia, histórico de variação do nível d'água e da pluma de fase livre são fundamentais para a interpretação correta dos resultados do teste. Ou seja, os testes de Tn são uma ferramenta a ser integrada na avaliação de áreas contaminadas com LNAPL, não devendo ser considerados de forma isolada, mas sim de forma combinada com outras informações da área. Ressalta-se que os valores obtidos são extremamente dependentes das condições ambientais no momento da realização do teste e, portanto, os resultados devem ser interpretados com cuidado e não de forma absoluta.

\section{AGRADECIMENTOS}

Ao Conselho Nacional de Desenvolvimento Científico e Tecnológico - CNPq pelo auxílio financeiro (processo 426953/2016-9). Os autores também agradecem o apoio das empresas Venner Ambiental, Diatech Ambiental, Envimax Consultoria e Estratégia Ambiental e HS Equipamentos e Serviços. Por fim, agradecem ao Núcleo de Apoio Técnico ao Ensino, Pesquisa e Extensão (NATEPE/ UNIFESP), do Instituto de Ciências Ambientais, Químicas e Farmacêuticas da Universidade Federal de São Paulo.

\section{CONTRIBUIÇÃO DOS AUTORES}

Garcia, M. V. C.: metodologia, investigação, curadoria de dados, análise formal, escrita primeira redação. Leite, E. C. P.: metodologia, investigação, curadoria de dados, análise formal, escrita primeira redação. Pede, M. A. Z.: conceituação, metodologia, supervisão. Shinzato, M. C.: conceituação, metodologia, supervisão, escrita - revisão e edição. Freitas, J. G. F.: conceituação, obtenção de financiamento, metodologia, supervisão, escrita — revisão e edição.

\section{REFERÊNCIAS}

AHMED, W.; PALMIER, C.; ATTEIA, O.; CLASS, H. Multiphase simulation model for validating the estimate of light non-aqueous phase liquids (LNAPL) transmissivity using bail-down test. Arabian Journal for Science and Engineering, v. 44, n. 6. p. 6.099-6.107, 2019. http://doi.org/10.1007/ s13369-018-3663-7

ALMEIDA, A. C. S.; SILVA, J. P. M; SIQUEIRA, A.; FREJLICH, J. Medida de viscosidade pelo método de Ostwald: um experimento didático. Revista Brasileira de Ensino de Física, v. 17, n. 4, p. 279-283, 1995.

AMERICAN PETROLEUM INSTITUTE (API). Soil and groundwater research bulletin number 18. Washington: American Petroleum Institute, 2003.

AMERICAN PETROLEUM INSTITUTE (API). API LNAPL Transmissivity Workbook: a tool for baildown test analysis - user guide. API Publication 4762. Washington: American Petroleum Institute, 2016.

ASTM INTERNATIONAL. ASTM E 2856-13: standard guide for estimation of LNAPL transmissivity. West Conshohocken, Pennsylvania: ASTM, 2013.

ATTEIA, O.; PALMIER, C.; SCHÄFER, G. On the influence of groundwater table fluctuations on oil thickness in a well related to an LNAPL contaminated aquifer. Journal of Contaminant Hydrology, v. 223, p. 103476, 2019. http://doi. org/10.1016/j.jconhyd.2019.03.008

AZIMI, R;: VAEZIHIR, A.; LENHARD, R. J:; HASSANIZADEH, M. Evaluation of LNAPL behavior in water table inter-fluctuate zone under groundwater drawdown condition. Water, v. 12, n. 9. p. 2.337, 2020. https://doi.org/10.3390/w12092337

BECKETT, G. D.; HUNTLEY, D. Guest editorial LNAPL transmissivity: a twisted parameter. Groundwater Monitoring \& Remediation, v. 35, n. 3, p. 20-24, 2015. https://doi.org/10.1111/gwmr.12116

BECKETT, G. D.; LUNDEGARD, P. D. Practically Impractical - The Limits of LNAPL Recovery and Relationship to Risk. In: Petroleum hydrocarbons \& organic chemicals in ground water: Prevention, detection, and remediation. Conferência, Houston, Proceedings..., Houston, United States, 1997.

CHARBENEAU, R. J.; JOHNS, R. T.; LAKE, L. W.; MCADAMS III, M. J. FreeProduct recovery of petroleum hydrocarbon liquids. Groundwater Monitoring and Remediation, v. 20, n. 3, p. 147-158, 2020. http://doi org/10.1111/j.1745-6592.2000.tb00280.x

COMPANHIA AMBIENTAL DO ESTADO DE SÃO PAULO (CETESB). Relação de Areas Contaminadas e Reabilitadas no Estado de São Paulo. CETESB, 2019. Disponível em: https://cetesb.sp.gov.br/areas-contaminadas/relacaode-areas-contaminadas/. Acesso em: 20 mar. 2020.

EBRAHIMI, F.; LENHARD, R. J.; NAKHAEI, M.; NASSERY, H. R. An approach to optimize the location of LNAPL recovery wells using the concept of a LNAPL specific yield. Environmental Science and Pollution Research, v. 26, n. 28, p. 28.714-28.724, 2019. https://doi.org/10.1007/s11356-019-06052-7

GATSIOS, E.; RINCON, J. G.; RAYNER, J. L.; MCLAUGHLAN, R. G.; DAVISG.B. LNAPL transmissivity as a remediation metric in complex sites under water table fluctuations. Journal of Environmental Management, v. 215, p. 40-48, 2018. https://doi.org/10.1016/j.jenvman.2018.03.026

GOOGLE. Região Metropolitana de São Paulo. Google Earth, 2020. Disponível em: https://goo.gl/maps/DphAeWZGGhn5HsoAA2O2O. Acesso em: 14 out. 2021.

HAWTHORNE, J. M.; KIRKMAN, A. Residual, Mobile and Migrating LNAPL. Applied NAPL Science Review. Demystifying NAPL Science for the Remediation Manager, v. 1, n. 10, p. 1-1, 2011. Disponivel em: http://naplansr. com/wp-content/uploads/2011/12/v1i1O.pdf. Acesso em: 10 mai. 2019.

HUNTLEY, D.; BECKETT, G. D. Persistence of LNAPL sources: relationship between riskreductionand LNAPL recovery.Journal of Contaminant Hydrology, v. 59, n. 1-2, p. 3-26, 2002. https://doi.org/10.1016/s0169-7722(02)00073-6 
ILLANGASEKARE, K. H.; JENSEN, K. H.; JAVANDEL, I.; MAYER, A. S. Migration and Distribution. In: MAYER, A. S; HASSANIZADEH, M. (Eds.). Soil and Groundwater Contamination: Non-aqueous Phase Liquids. Water Resources Monograph 17. Washington: American Geophysical Union, 2005. 224 p.

INSTITUTO DE PESQUISAS TECNOLÓGICAS DO ESTADO DE SÃO PAULO (IPT). Parecer Técnico no 7.703/00: Assessoria técnica para a regularização da atividade minerária na Estância Turística de Ribeirão Pires. São Paulo: IPT, 2020

INTERSTATE TECHNOLOGY \& REGULATORY COUNCIL (ITRC). Evaluating LNAPL remedial technologies for achieving project goals. Washington: ITRC, 2009

INTERSTATE TECHNOLOGY \& REGULATORY COUNCIL (ITRC). Light nonaqueous phase liquid (Inapl) site management: LCSM evolution, decision process, and remedial technologies. LNAPL-3. Washington: ITRC, 2018.

JENSEN, K. H.; FALTA, R. W. Fundamentals. In: MAYER, A. S; HASSANIZADEH, M. (Eds.). Soil and groundwater contamination: non-aqueous phase liquids. Water Resources Monograph 17. Washington: American Geophysical Union, 2005. 224 p.

KIMBALL, C. G.; HAWTHORNE, J. M; MENATTI, J. A.; ROUSSEAU, M. J. Light non-aqueous phase liquid transmissivity $(\mathrm{Tn})$ acceptance and use by the regulatory community. University of Tulsa, 2018. Disponivel em: https:// cese.utulsa.edu/wp-content/uploads/2018/03/Kimball-18.pdf. Acesso em: 15 mar. 2019.

KIRKMAN, A. J.; KOONS, B. Unifying NAPL drawdown and transmissivity testing in unconfined, confined, perched, and fractured settings using the Z-Factor and $\mathrm{MH}$ principles. Groundwater Monitoring and Remediation, v. 40, n. 1, p. 47-64, 2020. http://doi.org/10.1111/gwmr.12363

LENHARD, R. J.; RAYNER, J. L.; DAVIS, G. B. A practical tool for estimating subsurface LNAPL distributions and transmissivity using current and historical fluid levels in groundwater wells: Effects of entrapped and residual LNAPL. Journal of Contaminant Hydrology, v. 205, p. 1-11, 2017. https://doi. org/10.1016/j.jconhyd.2017.06.002
NEWELL, C. J.; ACREE, S. D.; ROSS, R. R.; HULLING, S. G. Ground water issue: light nonaqueous phase liquids. Washington: EPA, 1995, Disponivel em https://pdfs.semanticscholar.org/4386/10dOa94145d7c49be7af902af4fb62 91b4dc.pdf. Acesso em: 10 abr. 2019.

OOSTROM, M.; FALTA, R. W.; MAYER, A. S. Site characterization and Monitoring. In: MAYER, A. S; HASSANIZADEH, M. (Eds.), Soil and groundwater contamination: non-aqueous phase liquids. Water Resources Monograph 17. Washington: American Geophysical Union, 2005. 224 p.

PALMIER, C:; DODT, M.; ATTEIA, O. Comparison of oil transmissivity methods using bail-down test data. Groundwater Monitoring \& Remediation, v. 36, n 3, p. 73-83, 2016. http://doi.org/10.1111/gwmr.12173

PALMIER, C:; CAZALS, F.; ATTEIA, O. Bail-down test simulation at laboratory scale. Transport in Porous Media, v. 116, n. 2, p. 567-583, 2017. https://doi org/10.1007/s11242-016-0790-z

PARKER, J. C.; ISLAM. M. Inverse modeling to estimate LNAPL plume release timing. Journal of Contaminant Hydrology, v. 45, n. 3, p. 303-327, 202O. https://doi.org/10.1016/S0169-7722(00)00105-4

SIMON, J. A. Editor's perspective - LNAPL transmissivity: mobility is the new state-of-the-art cleanup metric. Remediation, v. 22, n. 3, p. 1-8, 2012. http://doi. org/10.1002/rem.21316

SOOKHAK LARI, K.; RAYNER, J. L.; DAVIS, G. B.; JOHNSTON, C. D. LNAPL recovery endpoints: Lessons learnt through modeling, experiments, and field trials. Groundwater Monitoring and Remediation, v. 40, n. 3, p. 21-29, 2020. https://doi.org/10.1111/gwmr.12400

TOMLINSON, D. W.; RIVETT, M. O.; WEALTHALL, G. P.; SWEENEY, R E. H. Understanding complex LNAPL sites: illustrated handbook of LNAPL transport and fate in the subsurface. Journal of Environmental Management, v. 204, p. 748-756, 2017. https://doi.org/10.1016/j. jenvman.2017.08.015

WINEGARDNER, D. L.; TESTA S. M. Restoration of contaminated aquifers: petroleum hydrocarbons and organic compounds. $2^{\mathrm{a}}$ ed. Boca Raton: CRC Press, 2000. 464 p. 